September 7, 2018

UCRHEP-T132

\title{
Anomalous commutator corrections to sum rules
}

\author{
JAVIER P. Muniain AND José WudKA \\ Department of Physics \\ University of California at Riverside \\ Riverside, California 92521-0413; U.S.A. \\ E-Mail address: jose.wudka@ucr.edu
}

\begin{abstract}
In this paper we consider the contributions of anomalous commutators to various QCD sum rules. Using a combination of the BJL limit with the operator product expansion the results are presented in terms of the vacuum condensates of gauge invariant operators. It is demonstrated that the anomalous contributions are no negligible and reconcile various apparently contradictory calculations.
\end{abstract}




\section{Introduction}

The use of canonical commutators in the evaluation of current algebra relations has produced many results whose effects are directly measurable. Still in many cases the canonical evaluation of the commutators is ill defined, as clearly exemplified by Schwinger's calculation ${ }^{1}$ of $\left\langle 0\left|\left[J^{0}(0, \mathbf{x}), J^{i}(0, \mathbf{y})\right]\right| 0\right\rangle$ for a conserved current $J^{\mu}$. If a fermionic current of the type $\bar{\psi} \gamma^{\mu} \psi$ is replaced in the above expression, and canonical commutation relations are used the above expression vanishes. In contrast, using general principles (such as Lorentz covariance and the absence of negative norm gauge-invariant states), the above vacuum expectation value is seen to be non-zero.

This fact has been used repeatedly (although somewhat sporadically) in the calculations of anomalous (i.e., non-canonical) contributions to various commutators, especially in the context of anomalous theories ${ }^{2}$. Similar effects have also been shown to modify the (canonically obtained) properties of the electroproduction sum rules ${ }^{3}$.

Faced with these problems in the canonical evaluation of commutation relations an alternative definition of the commutators was proposed by Bjorken and by Johnson and Low ${ }^{4}$. This definition preserves all the desirable features of the theory, is well defined and coincides with the canonical results whenever the latter are also well defined.

The starting point of the Bjorken-Johnson-Low (BJL) definition of the commutator of two operators $A$ and $B$ is the time ordered product $T(A B)$, presented as a function of the momentum transfer $p$. One then obtains the Laurent expansion of this operator in $p^{0}$ (the energy transfer). The term proportional to $1 / p^{0}$ is identified as (the Fourier transform of) the equal time commutator ${ }^{\# 1}$ Terms in this expansion containing positive powers of $p^{0}$ are associated with the covariantizing of the time-ordered product ${ }^{5}$ and can be ignored.

\#1 It is of course possible for this quantity to be divergent. 
The applications of this method have been largely restricted to perturbation theory (see however Refs. 6,7). On the other hand, many interesting applications of current algebra reside in the area where perturbation theory cannot be applied. In order to use the BJL definition in a wider range of situations we first note that the commutator is obtained by studying the relevant time-ordered products in the limit of large energy transfers, and, therefore, that an operator product expansion ${ }^{8}$ (OPE) is appropriate. The procedure which we follow is therefore to perform an OPE of the said time-ordered product, to then use renormalization group arguments to determine the high-energy behavior of the coefficient functions and thus extract the terms that contribute to the commutator. The result is then expressed in terms of the residues of the coefficient functions multiplied by the matrix elements of the local operators appearing in the $\mathrm{OPE}^{\# 2}$. In these calculations all symmetries of the theory are manifest, and so the resulting commutator will also respect them. A similar method was proposed by Crewther many years ago ${ }^{6}$ but was not developed significantly.

This paper is organized as follows. In the following section we describe the method in detail. Section 3 presents a comparison of the present method with some explicit perturbative calculations in $1+1$ dimensions. In sections 4 and 5 we consider the anomalous commutator modifications to the current algebra approach to the $U(1)_{A}$ problem. Section 6 presents some explicit calculations pertaining the general arguments presented in sections 4 and 5. Parting comments are presented in section 7 . The appendix contains the comparison of the present method with the results of perturbation theory for a $3+1$ dimensional model.

\#2 This is reminiscent of the results obtained using sum rules ${ }^{9}$. 


\section{Description of the method}

In this section we develop a useful technique which allows us to extract information about non-canonical contributions to equal time commutators without going through the lengthy steps of loop calculations involving triangle, box and even pentagon diagrams. This technique, which does not rely on perturbation theory, is based on the Bjorken-Johnson-Low definition of the equal time commutators and on the operator product expansion (OPE).

According to the BJL limit prescription, the definition of the commutator of two operators is obtained from the high energy behavior of Green's functions

$$
\begin{aligned}
& \lim _{p_{0} \rightarrow \infty} i p_{0} \int d^{n} x e^{-i p \cdot x}\langle\alpha|T A(x / 2) B(-x / 2)| \beta\rangle= \\
&=\int d^{n-1} x e^{i \mathbf{p} \cdot \mathbf{x}}\langle\alpha|[A(0, \mathbf{x} / 2), B(0,-\mathbf{x} / 2)]| \beta\rangle,
\end{aligned}
$$

where $p_{0}$ is the time-like component of the four momentum ${ }^{\# 3}$. The time ordered product $T$ is not Lorentz invariant and differs from the corresponding covariant Green's function by terms involving delta functions of $x_{0}$ and its derivatives (corresponding to a polynomial in $p_{0}$ in momentum space). If the left hand side of (2.1) is evaluated using covariant perturbation theory then all polynomials in $p_{0}$ should be dropped. The covariant time-ordered product will be denoted by $T^{*}$

Since we are interested in the large momentum behaviour of $\left\langle\alpha\left|T^{*} A B\right| \beta\right\rangle$, it is appropriate to express this object as a sum of local operators (OPE) where the coefficient functions summarize the $p_{0} \rightarrow \infty$ behaviour,

$$
\int d^{n} x e^{-i p \cdot x}\left\langle\alpha\left|T^{*} A(x / 2) B(-x / 2)\right| \beta\right\rangle=\sum_{r} c_{r}(p)\left\langle\alpha\left|\mathcal{O}_{r}\right| \beta\right\rangle,
$$

where the local operators $\mathcal{O}_{r}$ are evaluated at $x=0$. Taking the BJL limit of the

\#3 Double commutators can be defined using a straightforward generalization involving a double limit. 
previous expression we find

$$
\begin{aligned}
\int d^{n-1} x e^{i \mathbf{p} \cdot \mathbf{x}}\langle\alpha|[A(0, \mathbf{x} / 2), B(0,-\mathbf{x} / 2)]| \beta\rangle \\
=\sum_{r} \lim _{p_{0} \rightarrow \infty}\left[i p_{0} c_{r}(p)\right]\left\langle\alpha\left|\mathcal{O}_{r}\right| \beta\right\rangle,
\end{aligned}
$$

where, as mentioned above, all terms in the $c_{r}$ growing as a power of $p_{0}$ can be dropped.

It is well known that the matching of dimensions of the operators $T(A B)$ and $\mathcal{O}_{r}$ in the OPE must take into account the anomalous dimensions of these objects. This can be avoided provided the operators considered are renormalization group invariant, such as the trace of the energy momentum tensor or the fermion mass terms. Note that even if $A$ and $B$ are renormalization group invariant, the time ordered product $T(A B)$ need not have this property. In the most favorable cases the operators are renormalization group invariant and the canonical evaluation of the dimensions remains valid.

Another characteristic of the method is that the results are evaluated in terms of a set of unknown constants, the residues of the the coefficient functions $c_{r}$. For the applications which we consider this will not be a disadvantage: these constants multiply the matrix elements $\left\langle\alpha\left|\mathcal{O}_{r}\right| \beta\right\rangle$ which, in most cases cannot be evaluated to all orders in perturbation theory. Thus the final result will be given in terms of these "condensates" multiplied by the said constants. 


\section{Simple example}

As an application the previous remarks we consider a model containing fermions coupled to external non-Abelian gauge fields. We then choose $A=\mathcal{J}_{\mu}^{a}, B=\mathcal{J}_{\nu}^{b}$, where $\mathcal{J}$ denotes the right or left-handed, gauge invariant fermionic current, and $a, b$ denote color indices. Thus we consider

$$
\mathcal{T}_{\mu \nu}^{a b}=\int d^{n} x e^{-i p \cdot x} T^{*} \mathcal{J}_{\mu}^{a}(x / 2) \mathcal{J}_{\nu}^{b}(-x / 2)
$$

Similarly we define

$$
\mathcal{C}_{\mu \nu}^{a b}=\lim _{p^{0} \rightarrow \infty} i p_{0} \mathcal{T}_{\mu \nu}^{a b}
$$

(where the terms growing like a polynomial in $p_{0}$ are dropped as discussed in the previous section). In writing the operator expansion of this object we have to pick terms that have the same dimensionality and that posses same symmetries; in particular we can restrict ourselves to gauge invariant operators.

In $n$ dimensions $\mathcal{T} \sim(\text { mass })^{n-2}$, hence we can restrict ourselves to operators $\mathcal{O}_{r}$ of dimension equal or greater than $(n-2)$ on the right hand side of the OPE. We will consider here the $1+1$ dimensional case, leaving the $1+3$ case to the appendix, as it does not bring up any new ideas or physics, and it is somewhat more involved.

In $1+1$ dimensions $\mathcal{T}$ has canonical dimension zero. Moreover, the Dirac matrices satisfy $\gamma_{5} \gamma^{\mu}=\epsilon^{\mu \nu} \gamma_{\nu}$ which implies that we need only consider the vector currents, which we denote by $J_{\mu}^{a}$. The current is given explicitly by

$$
J_{\mu}^{a}(x, \epsilon)=\frac{i}{2} \bar{\psi}\left(x+\frac{\epsilon}{2}\right) \gamma_{\mu} T^{a}\left[\mathcal{P} \exp \left(\int_{x-\epsilon / 2}^{x+\epsilon / 2} A^{\sigma}(y) d y_{\sigma}\right)\right] \psi\left(x-\frac{\epsilon}{2}\right)+\text { h.c. }
$$

where $T^{a}$ denote the (anti-hermitian) group generators, \#4 "h.c" denotes the Hermitian conjugate, and $A^{\mu}=A_{a}^{\mu} T^{a}$ denotes the gauge field (the coupling constant is absorbed in the definition of $A$ ).

\#4 The conventions we use $\operatorname{are} \operatorname{tr}\left(T^{a} T^{b}\right)=-\delta_{a b},\left[T^{a}, T^{b}\right]=f_{a b c} T^{c}, \operatorname{tr} T^{a}\left\{T^{b}, T^{c}\right\}=-d_{a b c}$. 
The OPE of $\mathcal{T}$ (defined now for the vector currents by replacing $\mathcal{J} \rightarrow J$ in (3.1)) is then given by

$$
\mathcal{T}_{\mu \nu}^{a b}=c_{0 \mu \nu}^{a b} \mathbf{1}+c_{1 \mu \nu \rho}^{a b c}(p) J^{c \rho}+c_{2}^{a b} \underset{\mu \nu}{a b}(p) J^{\rho}+\cdots,
$$

where 1 denotes the unit operator, $J_{\rho}^{c}$ is defined in (3.3), $J_{\rho}$ denotes the singlet vector current $\sim \bar{\psi} \gamma_{\rho} \psi$, the ellipsis denote terms which will not contribute once the BJL limit is taken ${ }^{\# 5}$; the c-number functions $c_{r}$ must have dimension -1 for $r=1,2$ and 0 for $r=0$. Using the fact that $\mathcal{T}$ must be symmetric under $a \leftrightarrow b, \mu \leftrightarrow \nu, p \leftrightarrow-p$, the most general form of the coefficient functions is $\# 6$

$$
\begin{aligned}
c_{0 \mu \nu}^{a b}= & \delta_{a b}\left[\xi g_{\mu \nu}+\eta p_{\mu} p_{\nu} / p^{2}\right], \\
c_{1 \mu \nu \rho}^{a b c}(p)= & \frac{1}{p^{2}} d_{a b c}\left[\alpha\left(g_{\mu \rho} p_{\nu}-g_{\nu \rho} p_{\mu}\right)+\beta\left(\epsilon_{\mu \rho} p_{\nu}-\epsilon_{\nu \rho} p_{\mu}\right)+\gamma \epsilon_{\mu \nu} p_{\rho}\right] \\
& +\frac{1}{p^{2}} f_{a b c}\left[\frac{a}{2}\left(g_{\mu \rho} p_{\nu}+g_{\nu \rho} p_{\mu}\right)+\frac{b}{2}\left(\epsilon_{\mu \rho} p_{\nu}+\epsilon_{\nu \rho} p_{\mu}\right)\right. \\
& \left.+c g_{\mu \nu} p_{\rho}+d \frac{p_{\mu} p_{\nu} p_{\rho}}{p^{2}}\right], \\
c_{2 \mu \nu \rho}^{a b}(p)= & \frac{1}{p^{2}} \delta_{a b}\left[u\left(g_{\mu \rho} p_{\nu}-g_{\nu \rho} p_{\mu}\right)+v\left(\epsilon_{\mu \rho} p_{\nu}-\epsilon_{\nu \rho} p_{\mu}\right)+w \epsilon_{\mu \nu} p_{\rho}\right] .
\end{aligned}
$$

Denoting by $P$ the spatial component of the momentum the commutator for the vector currents, obtained by replacing $\mathcal{J} \rightarrow J$ in (3.1) and (3.2), is given by ${ }^{\# 7}$

$$
\begin{aligned}
& \frac{1}{i} \mathcal{C}_{00}^{a b}=f_{a b c}\left[(a+c+d) J_{0}^{c}+b J_{1}^{c}\right], \\
& \frac{1}{i} \mathcal{C}_{01}^{a b}=-\eta \delta_{a b} P-d_{a b c}\left[(\beta+\gamma) J_{0}^{c}+\alpha J_{1}^{c}\right]+\frac{1}{2} f_{a b c}\left(b J_{0}^{c}+a J_{1}^{c}\right) \\
& \quad \quad-\delta_{a b}\left[(v+w) J_{0}+u J_{1}\right], \\
& \frac{1}{i} \mathcal{C}_{11}^{a b}=-c f_{a b c} J_{0}^{c} .
\end{aligned}
$$

In the case where the theory has only right-handed couplings these relations im-

\#5 Note that the coefficient functions for the operators $\bar{\psi} T^{c} \psi, \bar{\psi} \psi, \bar{\psi} T^{c} \gamma_{5} \psi$ and $\bar{\psi} \gamma_{5} \psi$ will be of the form $\sim$ (mass parameter) $/ p^{2}$ and so will not contribute in the BJL limit.

\#6 The constants $\xi, \ldots, w$ can be evaluated perturbatively. We will not need their explicit expressions.

\#7 In our conventions $\epsilon^{01}=+1$. 
ply $^{\# 8} d=0$ and

$$
i\left[J_{R}^{a}(0, X / 2), J_{R}^{b}(0,-X / 2)\right]=\frac{i \eta}{2} \delta_{a b} \delta^{\prime}(X)-\frac{a+b}{2} f_{a b c} J_{R}^{c} \delta(X)
$$

This expression should be compared to those obtained in Ref. 10 which has the same form, except that $J_{R}$ is replaced by $A_{R}=A_{0}+A_{1}$. The discrepancy can be understood by following the procedure used in Ref. 10. What was done was to evaluate various matrix elements of the commutators and then to exhibit some local operators which have the same matrix elements. These operators are not unique, however. For example the matrix elements of $A_{R}$ and $J_{R}$ between the vacuum and the one gauge-boson state are proportional to each other in the zero momentum limit (the limit in the case of $J_{R}$ is taken symmetrically, first averaging over the direction of the momentum and then letting the magnitude go to zero). It is easy to see that the results of the diagrammatic calculations are consistent with those presented in Ref.10 when $A_{R}$ is replaced by $-2 \pi J_{R}$. It is in this sense that the above calculation in consistent with the explicit diagrammatic evaluation (up to the undetermined constants $\eta$ and $a+b$ which we do not evaluate at this point.) We also point out that the above expressions have the expected form when taking the matrix elements of the commutators for states containing fermions.

The above expressions of the anomalous commutators have the additional advantage of being manifestly gauge covariant. The terms proportional to $\delta(X)$ are generated by the matrix elements of the canonical contribution to the commutator. The only irreducible non-canonical contribution is the Schwinger term $\propto \delta_{a b} \delta^{\prime}(X)$. We shall see in the appendix that similar results hold in $3+1$ dimensions.

The above results can also be used for calculating the Schwinger term and seagull for the commutators under consideration. Writing the expressions using a

\#8 Note that in $1+1$ dimensions $J_{R \mu=0}^{a}=J_{R \mu=1}^{a}=J_{R}^{a}$. 
time-like unit vector $n^{5}$ we obtain for the commutator of two vector currents,

$$
\begin{aligned}
& \qquad\left[J_{R}^{a}(x / 2), J_{R}^{b}(-x / 2)\right] \delta(x \cdot n)=C_{\mu \nu}^{a b} \delta^{(2)}(x)+S_{\mu \nu}^{a b ; \alpha} \partial_{\alpha} \delta^{(2)}(x) \\
& \text { where } \quad C_{\mu \nu}^{a b}=-f_{a b c}\left(\left|g_{\mu \nu}\right| n \cdot J^{c}+\left|\epsilon_{\mu \nu}\right| n_{\rho} \epsilon^{\rho \sigma} J_{\sigma}^{c}\right) ; \\
& S_{\mu \nu}^{a b ; \alpha}=\delta^{a b} \eta\left|\epsilon_{\mu \nu}\right| n_{\rho} \epsilon^{\rho \alpha}
\end{aligned}
$$

From this it follows that the corresponding seagull vanishes.

Thus the method is seen to work to lowest order in perturbation theory. The disadvantage is that the final result is expressed in terms of a few unknown constants which, if required, can only be obtained doing detailed calculations. Moreover, for higher orders in perturbative calculations the anomalous dimensions of the various operators must be taken into account. We have seen that the apparently gauge variant results obtained in the literature can be re-interpreted as generated by the canonical terms in the commutator.

\section{Current algebra and the $\mathrm{U}(1)$ problem.}

In this section we will consider the effects of anomalous commutators in the study of the $U(1)_{A}$ problem. In this area the results obtained using instanton

calculations $^{11}$ were criticized $^{12}$ on the basis of certain inconsistencies which arise when the commutators involved are evaluated using canonical expressions. We will see that the relations derived in Ref. 12 are in general modified due to the anomalous terms in the commutators; this point is also made in Refs. 13 and 14 where it is noted that configurations carrying topological charge affect the pion decay constant Reference 12 also points out several apparent contradictions concerning the periodicity of the $\theta$ angle within the instanton and the canonical approaches. This problem was investigated in Refs. 13 and 14 and found to be rooted in a mis-application of the index theorem for which there are subtleties connected with spontaneous symmetry breaking. 
These modifications are sufficient to explain the differences between the two approaches.

To specify the notation we denote by $J_{5}^{\mu}$ the gauge-invariant anomalous current which, in the presence of $\ell$ massless flavors, satisfies

$$
\partial_{\mu} J_{5}^{\mu}=\ell \nu ; \quad \nu=\frac{g^{2}}{16 \pi^{2}} F \cdot \tilde{F}
$$

The charge associated with this current is denoted by $Q_{5}=\int d^{3} x J_{5}^{0}$.

In the effective lagrangian description the effects of an instanton (respectively, an anti-instanton) localized at $x$ is described by a potential ${ }^{14} U_{\mathrm{a}}$ (respectively, $U_{\mathrm{a}}^{*}$ ) with the identification ( $\ell$ is the number of light quark flavors)

$$
\ell \nu(x) \leftrightarrow 8 \operatorname{Im} U_{\mathrm{a}}(x)
$$

where $\nu$ is defined in (4.1). The potential $U_{\mathrm{a}}$ is proportional to a quark-determinant operator involving all light flavors ${ }^{11,14}$.

The problem arises because $U_{\mathrm{a}}$ has chirality $2 \ell$ and so the right hand side of (4.2) has non-trivial commutator with the axial charge (as constructed in the effective theory). In contrast, $\nu$ apparently commutes with $Q_{5}$, thus raising questions about the above identification. This contradiction can be solved by using the BJL definition of the commutator between $\nu$ and $J_{5}^{\mu}$.

As a fist step we consider, for example, the vacuum correlator

$$
\int d^{4} x e^{-i p \cdot x}\left\langle 0\left|T^{*} \nu(x / 2) J_{5}^{\mu}(-x / 2)\right| 0\right\rangle=\sum_{r} c_{r}^{\mu}(p)\left\langle 0\left|\mathcal{O}_{r}\right| 0\right\rangle .
$$

The lowest dimensional (non-trivial) operator that contributes to the right hand side is the trace of the energy-momentum tensor which we denote by $\Theta$. We expect the commutator to be a renormalization group invariant quantity; in this case the coefficient function associated with $\Theta$ will have the form $c_{\Theta}^{\mu}(p)=\bar{c}_{\Theta} p^{\mu} / p^{2}$. 
We hasten to point out that the OPE is valid only for large $p$ and so one cannot interpret this form of $c_{\Theta}^{\mu}(p)$ as corresponding to a massless pseudoscalar excitation. We then obtain

$$
\int d^{4} x e^{i \mathbf{p} \cdot \mathbf{x}}\left\langle 0\left|\left[\nu(0, \mathbf{x} / 2), J_{5}^{0}(0,-\mathbf{x} / 2)\right]\right| 0\right\rangle=i \bar{c}_{\Theta}\langle 0|\Theta| 0\rangle
$$

Since we expect both $\bar{c}_{\Theta}$ and $\langle 0|\Theta| 0\rangle$ to be non-vanishing, it follows that the commutator of $\nu$ with $Q_{5}$ is non-trivial also within the context of $\mathrm{QCD}^{\# 9}$.

When quark masses are included the above equation is modified since more operators become available. Specifically, one can include on the right hand side of (4.3) a term containing the operator $D$, where

$$
D=2 \sum_{f} m_{f} \bar{q}_{f} q_{f}
$$

( $m_{f}$ and $q_{f}$ denote the mass and field associated with the quark of flavor $f$ ). In this case the corresponding coefficient function in the OPE (4.3) tales the form $c_{D}^{\mu}=\bar{c}_{D} p^{\mu} / p^{2}$, and (4.4) becomes

$$
\int d^{4} x e^{i \mathbf{p} \cdot \mathbf{x}}\left\langle 0\left|\left[\nu(0, \mathbf{x} / 2), J_{5}^{0}(0,-\mathbf{x} / 2)\right]\right| 0\right\rangle=i \bar{c}_{\Theta}\langle 0|\Theta| 0\rangle+i \bar{c}_{D}\langle 0|D| 0\rangle .
$$

As we will see below, $\bar{c}_{\Theta, D}$ do not in general vanish. Hence the commutator between $\nu$ and $Q_{5}$ receives non-canonical contributions. Model calculations ${ }^{14}$ also show that the expression for the said commutator acquires a non-canonical piece proportional to $\langle 0|\Theta| 0\rangle$. The right hand side of (4.6) should vanish for massless quarks in the $\mathbf{p} \rightarrow 0$ limit; this is verified within a specific model in section 6 .

\footnotetext{
\#9 A straightforward perturbative calculation shows that, at least to one loop, $\bar{c}_{\Theta} \neq 0$; see below.
} 


\section{Anomalous Ward identities}

In the previous section we remarked that the operator $\nu$ can have non-zero commutator with the gauge invariant axial current $J_{5}^{\mu}$; in particular $\left[\nu, Q_{5}\right] \neq$ 0 . These results are supported by a straightforward application of the effective lagrangian proposed in Ref. 14. It is of course possible for the constant $\bar{c}_{\Theta}$ (and $\bar{c}_{D}$ is $m_{f} \neq 0$ ) to vanish, but this would not be consistent with the effective lagrangian approach. We also point out that $\nu$ will mix under renormalization with operators which have non-zero chirality.

Should the above commutator be different form zero, the anomalous Ward identities will be modified. Consider then a gauge invariant operator $\mathcal{O}$ and define

$$
\Pi_{\mu}^{(\mathcal{O})}(p)=\int d^{4} x e^{-i p \cdot x}\left\langle 0\left|T J_{5 \mu}(x) \mathcal{O}(0)\right| 0\right\rangle
$$

The requirement that there be no light isosinglet pseudoscalars ${ }^{12,9}$ implies that $p \cdot \Pi^{(\mathcal{O})}$ will vanish as $p \rightarrow 0$. It follows that, by the definition of the $T$ symbol,

$$
\begin{aligned}
0 & =\int d^{4} x\left\langle 0\left|T \partial \cdot J_{5}(x) \mathcal{O}(0)\right| 0\right\rangle+\left\langle 0\left|\left[Q_{5}, \mathcal{O}(0)\right]\right| 0\right\rangle \\
& =\int d^{4} x\langle 0|T \Delta(x) \mathcal{O}(0)| 0\rangle+\int d^{4} x\langle 0|T \ell \nu(x) \mathcal{O}(0)| 0\rangle+\left\langle 0\left|\left[Q_{5}, \mathcal{O}(0)\right]\right| 0\right\rangle
\end{aligned}
$$

where

$$
\Delta=2 i \sum_{f=1}^{\ell} m_{f} \bar{q}_{f} \gamma_{5} q_{f}
$$

and $\nu$ is defined in (4.1); we have assumed that the anomaly equation, $\partial \cdot J_{5}=$ $\Delta+\ell \nu$, is an operator identity.

Now, following Refs. 12 and 9, we consider (5.2) for the cases $\mathcal{O}=\ell \nu$ and $\mathcal{O}=$ $\Delta$; cancelling the correlator of $\Delta$ and $\nu$ which appears in both these expressions 
we obtain

$\ell^{2} \int d^{4} x\langle 0|T \nu(x) \nu(0)| 0\rangle=\left\langle 0\left|\left[Q_{5}, \Delta(0)-\ell \nu(0)\right]\right| 0\right\rangle+\int d^{4} x\langle 0|T \Delta(x) \Delta(0)| 0\rangle$.

The $T$-product on the right hand side equals the corresponding $T^{*}$ product. This is because the approach described in section 2 shows that there are no Schwinger terms in the equal-time commutator of $\Delta(x)$ and $\Delta(y)$; the corresponding seagull is therefore zero ${ }^{5}$. The commutator $\left[Q_{5},, \Delta\right]$ is proportional to $D=2 \sum m \bar{q} q ;$ we will write

$$
i\left[Q_{5}, \Delta\right]=2(1+\delta) D, \quad D=2 \sum_{f=1}^{\ell} m_{f} \bar{q}_{f} q_{f}
$$

where $\delta=0$ if the commutator is evaluated canonically. Finally we have $i\left[Q_{5}, \nu\right]=$ $\bar{c}_{\Theta} \Theta+\bar{c}_{D} D$, where $\Theta$ denotes the trace of the energy-momentum tensor. Thus we obtain, for the case of two light flavors $(\ell=2)$,

$2 i \int d^{4} x\langle 0|T \nu(x) \nu(0)| 0\rangle=\left(\delta-\bar{c}_{D}\right)\langle 0|D| 0\rangle-\bar{c}_{\Theta}\langle 0|\Theta| 0\rangle-4 f_{\pi} m_{\pi}^{2} \frac{m_{u} m_{d}}{\left(m_{u}+m_{d}\right)^{2}}$.

The fist two terms come from the anomalous commutators, while the last term is generated by the canonical commutator and the $T \Delta \Delta$ contributions as evaluated in Ref. 9.

The above calculation show that in general we can expect deviations form the canonical expression for the dependence of measurable quantities on the CPviolating parameter $\theta$. It is of course possible for the non-canonical terms to vanish, still explicit perturbative calculations and effective-lagrangian arguments favor $\bar{c}_{\Theta, D}, \delta \neq 0$. Since the dependence on $\theta$ disappears from all physical observables when one fermion is massless, we expect $\bar{c}_{\Theta}\langle 0|\Theta| 0\rangle-\delta\langle 0|D| 0\rangle$ to vanish when any quark mass is zero. We can then write

$$
i \int d^{4} x\langle 0|T \nu(x) \nu(0)| 0\rangle=-2 f_{\pi}^{2} m_{\pi}^{2}(1-\lambda) \frac{m_{u} m_{d}}{\left(m_{u}+m_{d}\right)^{2}},
$$

for some constant $\lambda$. The conditions under which $\lambda=1$ (or even if this is at all 
possible) cannot be determined using general arguments. We will see below that low-energy models of the strong interactions predict $1-\lambda=O(1)$ (see below) so that the estimates of physical quantities on the trong $\mathrm{CP}$ angle $\theta$ are altered only by a factor of $O(1)$ (except, of course, in the case $\lambda=1$ ).

In the following section we present several calculations where the various coefficients and vacuum condensates are evaluated within explicit models.

\subsection{Explicit CALCULATions}

In this section we present several computations. We have evaluated the OPE coefficients $\bar{c}_{\Theta}, \bar{c}_{D}$ and $\delta$ to lowest order in QCD; we also evaluate the condensates $\langle 0|\Theta| 0\rangle$ and $\langle 0|D| 0\rangle$ in a chiral model of the strong interactions.

\section{Perturbative calculations}

We first consider the calculation of $c_{\theta}^{\mu}$. The OPE of the product $T^{*} J_{5}^{\mu} \nu$ contains, to lowest order, three operators: $D$ defined in (4.5), $\Theta_{\mu \nu}$, the energymomentum tensor, and $\Theta$, its trace. For the calculation at hand we evaluate the matrix element between the vacuum and a two gluon state, the relevant graphs are presented in figure 1.

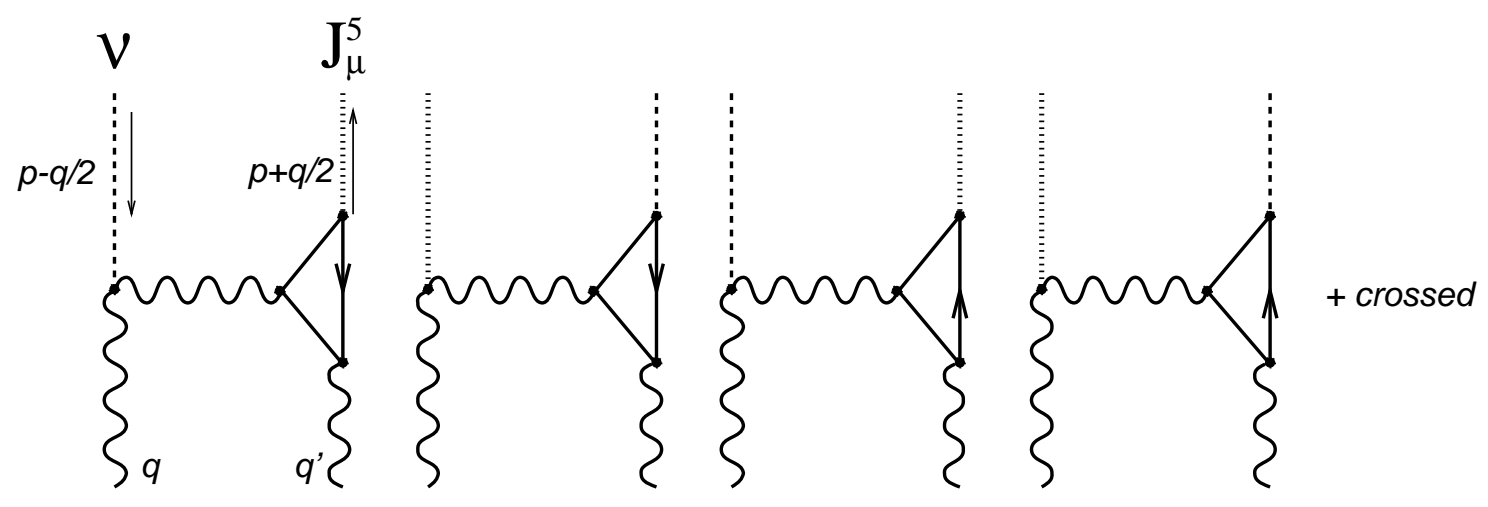

Fig. 1 Lowest order diagrams contributing to the OPE coefficient of $\Theta$ in the operator $T^{*} J_{\mu}^{5} \nu$.

In order to obtain the OPE coefficients in the $p \rightarrow \infty$ limit we need only 
consider the diagrams contracted with the momentum carried by the axial current, i.e., we multiply each graph by $(p+q / 2)^{\mu}$ (the momentum of the axial current) and contract the index $\mu$. A simple calculation using

$$
\Theta=\frac{\beta(\alpha)}{4 \alpha}\left(F_{\mu \nu}^{a}\right)^{2}+\frac{1}{2}\left(1+\gamma_{m}\right) D
$$

where $\beta$ denotes the beta function for $\alpha=g^{2} / 4 \pi$ and $\gamma_{m}$ the mass anomalous dimension. Assuming the presence of $\ell$ fermions we obtain

$$
\bar{c}_{\Theta}=\ell \frac{\alpha^{3}}{\pi^{2} \beta(\alpha)} ; \quad \beta(\alpha)=-\frac{11-2 \ell / 3}{2 \pi} \alpha^{2}+O\left(\alpha^{3}\right)
$$

We now consider $\bar{c}_{D}$ which can be extracted from the matrix element of $T \nu J_{5}^{\mu}$ between the vacuum and a two fermion state. It is easy to see, however, that the graphs for this matrix element are all $O\left(\alpha^{2}\right)$; in contrast $\bar{c}_{\Theta}=O(\alpha)$. The OPE for $T \nu J_{5}^{\mu}$ contains both $\Theta$, defined in (5.8), and $D$. It follows that the $O(\alpha)$ term in $\bar{c}_{\Theta}$ should be cancelled by a similar term in $\bar{c}_{D}$, hence

$$
\bar{c}_{D}=\frac{1}{2} \bar{c}_{\Theta}
$$

Finally we calculate $\delta$ by evaluating the matrix element of $T^{*} \Delta J_{\mu}^{5}$ between the vacuum and a two fermion state. We skip for brevity the description of the graphs; the final result is

$$
\delta=-\frac{9}{4} \frac{\alpha}{\pi}
$$

to first order in $\alpha$.

these calculations show that, at lest to first order in perturbation theory, all the anomalous coefficients are finite and non-vanishing as claimed previously. 


\subsection{Model Calculations}

In order to obtain estimates of the condensates $\langle 0|\Theta| 0\rangle$ and $\langle 0|D| 0\rangle$ we consider a chiral model which obeys the same symmetries as QCD. In order to generate Green's functions involving $\nu$ we modify the QCD lagrangian by adding a term $\theta \nu / 2$, with $\theta$ an external source (for details see Ref. 15). The Lagrangian takes the form ${ }^{15}$

$$
\mathcal{L}=-V_{0}+V_{1} \operatorname{tr} \partial_{\mu} U^{\dagger} \partial^{\mu} U+\left(V_{2} \operatorname{tr} M U+\text { H.c }\right)+V_{3} \partial \phi_{0} \cdot \partial \theta+V_{4}(\partial \theta)^{2}
$$

where $V_{i}=V_{i}\left(\phi_{0}+\theta\right), V_{i \neq 2}$ real, and $V_{i}(\alpha)=V_{i}^{*}(-\alpha)$. The meson field, denoted by $U$, belongs to the unitary field $U(\ell)$; we will write $U=\exp \left(i \phi_{0} / \ell\right) \Sigma$ with $\Sigma \in S U(\ell)$. For $\ell=3 \Sigma$ describer the pseudoscalar meson octet and $\phi_{0}$ describes the pseudoscalar isosinglet (the $\left.\eta^{\prime}\right)$.

The field $\Sigma$ describes the usual pseudoscalar meson multiplet (under $S U(\ell)$ flavor); $\phi_{0}$ describes the pseudoscalar singlet (i.e., the $\eta$ for $\ell=2$, and the $\eta^{\prime}$ for $\ell=3)$.

This model is an accurate representation of QCD at low momentum transfers, so we will not use it in obtaining the BJL definition of the commutators (which involve the $p^{0} \rightarrow \infty$ limit). We can, however, use this model to evaluate the condensates $\langle 0|\Theta| 0\rangle$ and $\langle 0|D| 0\rangle$ and the low momentum limit of $T^{*} \nu \nu$. We will, for simplicity, work in the $S U(\ell)$ symmetric limit where $M=m \mathbf{1}$.

To lowest order in a momentum expansion the correlator $\langle 0|T| 0\rangle^{*} \nu \nu$ can be obtained by replacing $U$ in $\mathcal{L}$ by the solution to the classical equations of motion ${ }^{15}$. A simple calculation shows that the lagrangian then takes the form

$$
\overline{\mathcal{L}}=\frac{1}{2} \theta \mathcal{K} \theta+\text { const.; } \quad \mathcal{K}=-2\left[V_{4}(0)+\text { inv } \ell V_{1}(0)-V_{3}(0)\right] \partial_{\mu} \partial^{\mu}-\frac{2 m}{\ell} V_{2}(0)
$$

which shows that the vacuum correlator $\left\langle 0\left|T^{*} \nu \nu\right| 0\right\rangle$ is proportional to $m$ in the limit of zero momentum transfer.

The various condensates can also be evaluated within this model. From Ref. 15 we get

$$
\langle 0|D| 0\rangle=-2 \ell m_{\pi}^{2} f_{\pi}^{2} ; \quad\langle 0|\Theta| 0\rangle=4 V_{0}(0)-4 \ell m_{\pi}^{2} f_{\pi}^{2},
$$

where $m_{\pi}$ denotes the (degenerate) meson mass and $f_{\pi}$ the corresponding decay constant. Perturbative calculations suggest that $\bar{c}_{\Theta}$ remains non-zero as $m \rightarrow 0$, 
hence consistency of the OPE with the above expressions for $T^{*} \nu \nu,\langle 0|D| 0\rangle$ and $\langle 0|\Theta| 0\rangle$ requires $V_{0}(0)$ to vanish as $m \rightarrow 0$. It follows that both condensates $\langle 0|D| 0\rangle,\langle 0|\Theta| 0\rangle$ vanish in this limit. This result justifies the claims made at the end of the previous section concerning the behaviour of the condensates in the zero mass limit. Similar results are obtained using the (closely related) model of Ref. 14

Within this model $i \int d^{4} x\left\langle 0\left|T^{*} \nu \nu\right| 0\right\rangle=-\frac{1}{2}\left(m_{\pi} f_{\pi}\right)^{2}$ which corresponds to $\lambda=$ 0 in (5.7). Thus the possibility of having $\lambda=1$ and a dynamical cancellation of the dependence on the strong $\mathrm{CP}$ angle is not realized, at least within this model.

\section{Conclusions}

In this paper we considered the BJL definition for the commutators and applied it in conjunction with the operator product expansion. The method can be applied both in the perturbative and non-perturbative regimes. As an application of the first case we considered the anomalous commutator between chiral currents in $1+1$ and $3+1$ dimensions. We showed that in this case the results in the literature can be re-interpreted to yield a gauge invariant expression for the commutators. The method here proposed is consistent with these results.

In the non-perturbative regime we considered the current algebra relations between the instanton number density $\nu$ and the gauge-invariant anomalous axial charge. We showed that, in general, this commutator is non-vanishing, in accordance with the results obtained using instanton calculations. We also noted that this conclusion is based on the no-trivial chiral transformation properties of the instanton density and this leads to some modifications of the expressions resulting from the anomalous Ward identities.

The method requires some knowledge about behaviour of the coefficient functions (which appear in the OPE) at large momentum transfers $p$. In asymptotically free theories this is available via the renormalization group. The final results are expressed in terms of the residues of the coefficient functions (i.e., the constant multiplying the term behaving as $1 / p_{0}$ ) and of the matrix elements of various local operators (the "condensates"). These quantities can be evaluated explicitly within perturbation theory; in the non-perturbative regime the condensates cannot be evaluated explicitly but can be used to parametrize the results.

Whereas the OPE coefficients can be evaluated perturbatively to any desired order of accuracy, the condensates are not calculable in this manner; for these quantities effective models must be considered. Unfortunately the effective theories 
are valid only at small momenta and this implies that the $p^{0} \rightarrow \infty$ limit of the OPE coefficient functions cannot be accurately evaluated using these theories. Explicit calculations verify several claims made on general grounds: there are non-trivial non-canonical contributions to the commutators. These contributions can be used to reconcile the operator and instanton approaches to the $U(1)_{A}$ problem.

Acknowledgements:

J.W. would like to thank R. Akhoury and R. Jackiw for useful comments. This work was supported in part through funds provided by the Department of Energy under contract DE-FG03-94ER40837.

\section{APPENDIX}

In this appendix we consider the more complicated case of the OPE of the two current correlator in $3+1$ dimensions. In order to keep the discussion at a manageable level we will consider the case

$$
\mathcal{T}_{\mu \nu}^{a b}(p)=\int d^{4} x e^{-i p \cdot x} T^{*} J_{\mu 5}^{a}(x / 2) J_{\nu}^{b}(-x / 2)
$$

Now $\mathcal{T}$ has mass dimension $=2$, leading us to a more complicated OPE. The relevant terms are $\# 10$

$$
\begin{aligned}
\mathcal{T}_{\mu \nu}^{a b}(p)=\frac{1}{p^{2}} & {\left[u_{1}^{a b c} p^{\alpha} p_{\nu} \tilde{F}_{\mu \alpha}^{c}+u_{2}^{a b c} p^{\alpha} p_{\mu} \tilde{F}_{\nu \alpha}^{c}\right.} \\
& +\left(u_{3}^{a b c} \mathcal{D}_{\mu} \tilde{F}_{\nu \alpha}^{c}+u_{4}^{a b c} \mathcal{D}_{\nu} \tilde{F}_{\mu \alpha}^{c}+u_{5}^{a b c} \mathcal{D}_{\alpha} \tilde{F}_{\mu \nu}^{c}\right) p^{\alpha} \\
& +u_{6}^{a b c} J_{5 \mu}^{c} p_{\nu}+u_{7}^{a b c} J_{5 \nu}^{c} p_{\mu}+u_{8}^{a b c} J^{c \rho} p^{\sigma} \epsilon_{\mu \nu \rho \sigma} \\
& \left.+\delta_{a b}\left(v_{1} J_{5 \mu} p_{\nu}+v_{2} J_{5 \nu} p_{\mu}+v_{3} J^{\rho} p^{\sigma} \epsilon_{\mu \nu \rho \sigma}\right)\right]
\end{aligned}
$$

where

$$
u_{i}^{a b c}=u_{i}^{(f)} f_{a b c}+u_{i}^{(d)} d_{a b c}
$$

(note that $\mathcal{D}^{\alpha} \tilde{F}_{\alpha \mu}=0$ due to the Bianchi identities).

\#10 We use the following conventions: $\tilde{F}_{\mu \nu}^{c}=\frac{1}{2} \epsilon_{\mu \nu \rho \sigma} F_{c}^{\rho \sigma}$, with $F_{\rho \sigma}^{c}=\partial_{\rho} A_{\sigma}^{c}-\partial_{\sigma} A_{\rho}^{c}+f_{a b c} A_{\rho}^{a} A_{\sigma}^{b}$.

The covariant derivative is given by $\mathcal{D}_{\mu} \tilde{F}_{\rho \sigma}^{c}=\partial_{\mu} \tilde{F}_{\rho \sigma}^{c}+f_{d e c} A_{\mu}^{d} \tilde{F}_{\rho \sigma}^{e}$. 
Applying the BJL limit to the previous expression, we obtain

$$
\begin{aligned}
& \lim _{p_{0} \rightarrow \infty} p_{0} \mathcal{T}_{\mu \nu}^{a b}(p)=i \int d^{3} x e^{i \mathbf{p} \cdot \mathbf{x}}\left[J_{\mu 5}^{a}(0, \mathbf{x} / 2), J_{\nu}^{b}(0,-\mathbf{x} / 2)\right] \\
&=\left[u_{1}^{a b c}\left(g_{\nu i} \tilde{F}_{\mu 0}^{c}+g_{\nu 0} \tilde{F}_{\mu i}^{c}\right)+u_{2}^{a b c}\left(g_{\mu i} \tilde{F}_{\nu 0}^{c}+g_{\mu 0} \tilde{F}_{\nu i}^{c}\right)\right] p^{i} \\
&+\left(u_{3}^{a b c} \mathcal{D}_{\mu} \tilde{F}_{\nu 0}^{c}+u_{4}^{a b c} \mathcal{D}_{\nu} \tilde{F}_{\mu 0}^{c}+u_{5}^{a b c} \mathcal{D}_{0} \tilde{F}_{\mu \nu}^{c}\right) \\
&+u_{6}^{a b c} J_{5 \mu}^{c} g_{\nu 0}+u_{7}^{a b c} J_{5 \nu}^{c} g_{\mu 0}+u_{8}^{a b c} J^{c \rho} \epsilon_{\mu \nu \rho 0} \\
&+ \delta_{a b}\left[v_{1} J_{5 \mu} g_{\nu 0}+v_{2} J_{5 \nu} g_{\mu 0}+v_{3} J^{\rho} \epsilon_{\mu \nu \rho 0}\right] .
\end{aligned}
$$

The equal time commutator for the $\mu=\nu=0$ case is given by

$$
\begin{aligned}
i\left[J_{05}^{a}(0, \mathbf{x} / 2), J_{0}^{b}(0,-\mathbf{x} / 2)\right]= \\
=-i\left(u_{1}^{a b c}+u_{2}^{a b c}\right) \mathbf{B}^{c} \cdot \nabla \delta(\mathbf{x}) \\
+\left[\left(u_{6}^{a b c}+u_{7}^{a b c}\right) J_{50}^{c}+\delta_{a b}\left(v_{1}+v_{2}\right) J_{50}\right] \delta(\mathbf{x}),
\end{aligned}
$$

where $\mathbf{B}^{c}$ denotes the chromo-magnetic field, $B^{i}=\tilde{F}_{0 i}$. The commutator for the space component of the vector current and the time component of the axial current is

$$
\begin{aligned}
i\left[J_{05}^{a}(0, \mathbf{x} / 2), \mathbf{J}^{b}(0,-\mathbf{x} / 2)\right]= & \\
= & i u_{2}^{a b c} \mathbf{E}^{c} \times \nabla \delta(\mathbf{x}) \\
& +\left[\left(u_{3}^{a b c}-u_{5}^{a b c}\right) \mathcal{D}_{0} \mathbf{B}^{c}+u_{7}^{a b c} \mathbf{J}_{5}^{c}+\delta_{a b} v_{2} \mathbf{J}_{5}\right] \delta(\mathbf{x})
\end{aligned}
$$

where $\mathbf{E}^{c}$ denotes the chromo-electric field, $E^{i}=F_{0 i}$.

These results are, as in the $1+1$ dimensional case, manifestly gauge covariant. We have verified that they are consistent with the explicit loop calculations presented in ${ }^{10}$; for example the two-gauge-boson matrix elements of $i d_{c b e} f_{\text {ead }} \tilde{F}_{d}^{0 k} A_{k}^{c}$ and $8 \pi^{2} f_{a b c} J_{R \mu=0}^{c}$ coincide.

These results can be used to calculate the corresponding Schwinger terms and covariantizing seagulls. Following the procedure described in Ref. 5 we obtain $\left[J_{\mu 5}^{a}(x / 2), J_{\nu}^{b}\right] \delta(n \cdot x)=C_{\mu \nu}^{a b} \delta^{(4)}(x)+S_{\mu \nu ; \alpha}^{a b} \partial^{\alpha} \delta^{(4)}(x)$ where the Schwinger term 
equals (the computation is straightforward and only the results will be presented)

$$
S_{\mu \nu ; \alpha}^{a b}=-\left[u_{1}^{a b c}\left(g_{\nu \alpha} \tilde{F}_{\mu \beta}^{c}+g_{\nu \beta} \tilde{F}_{\mu \alpha}^{c}\right)+u_{2}^{a b c}\left(g_{\mu \alpha} \tilde{F}_{\nu \beta}^{c}+g_{\mu \beta} \tilde{F}_{\nu \alpha}^{c}\right)\right] n^{\beta}
$$

and the corresponding seagull is

$$
\tau_{\mu \nu}^{a b}=-\left(u_{1}^{a b c} n_{\nu} \tilde{F}_{\mu \alpha}^{c}+u_{2}^{a b c} n_{\mu} \tilde{F}_{\nu \alpha}^{c}\right) n^{\alpha}
$$

We also remark that (again following the procedure described in Ref. 5) when both currents are conserved the requirement that the $T^{*}$ product be Lorentz covariant implies $u_{3}^{a b c}=u_{5}^{a b c}, u_{1}^{a b c}+u_{4}^{a b c}+u_{5}^{a b c}=0$ and $u_{6}^{a b c}=u_{7}^{a b c}=v_{1}=v_{2}=0$. Finally we note that the conditions under which the Schwinger terms cancel against the seagull contributions to the Ward identities is simply $u_{1}^{a b c}+u_{2}^{a b c}=0$.

\section{REFERENCES}

1. J. Schwinger Phys. Rev. Lett. 3, 269 (1959). T. Goto and I. Imamura, Prog. Theo. Phys. 14, 196 (1955).

2. S. Jo, Nucl. Phys. B259, 616 (1985); Phys. Rev. D35, 3179 (1987). K. Fujikawa, Phys. Lett. B171, 424 (1986), Phys. Lett. B188, 115 (1987). M. Kobayashi et al., Nucl. Phys. B273, 607 (1986). S.R. Gautam and D.A. Dicus, Phys. Rev. D12, 3310 (1975). J.M. Cornwall and R.E. Norton, Phys. Rev. 177, 2584 (1969).

3. R. Jackiw and J. Preparata, Phys. Rev. Lett. 22, 975 (1969), Phys. Rev. Lett. 22, 1162 (1969) (errata); Phys. Rev. 185, 1748 (1969). S.L. Adler and Wu-Ki Tung, Phys. Rev. Lett. 22, 978 (1969); Phys. Rev. D1, 2846 (1970).

4. J.D. Bjorken, Phys. Rev. 148, 1467 (1966). K. Johnson and F.E. Low Prog. Theo. Phys. Suppl. 37-38, 74 (1966).

5. For a reference see R. Jackiw in Lectures on Current Algebra and Its Applciations, by S.B. Treiman, R. Jackiw and D. J. Gross, (Princeton 1972). 
6. R.J. Crewther, Phys. Rev. Lett. 28, 1421 (1972).

7. S.L. Adler et al., Phys. Rev. D6, 2982 (1972)

8. K.G. Wilson, Phys. Rev. 179, 1499 (1969).

9. M. A. Shifman, A. I. Vainshtein and V. I. Zakharov, Nucl. Phys. B166, 493 (1980).

10. S. Jo, Ref. 2.

11. G. 't Hooft, Phys. Rev. Lett. 37, 8 (1976); Phys. Rev. D14, 3432 (1976).

12. R.J. Crewther, Phys. Lett. B70, 349 (1977). G.A. Christos, Phys. Rep. 116, 251 (1984).

13. E. Mottola, Phys. Rev. D21, 3401 (1980).

14. G. 't Hooft Phys. Rep. 142, 357 (1986)

15. J. Gasser and H. Leutwyler, Nucl. Phys. B250, 465 (1985); Ann. Phys. 158, 142 (1984). J. Gasser et al., Nucl. Phys. B3, 0 (7) (1988) 779. A. Pich, lectures presented at the V Mexican School of Particles and Fields, Guanajuato, México, Dec. 1992. 